\title{
Evaluación de la eficiencia del coagulante SuperFloc SD 2080 frente al cloruro férrico en procesos de clarificación de agua
}

\section{Evaluation of the efficiency of the SuperFloc SD 2080 coagulant versus ferric chloride in water clarification processes}

\author{
Anel Alcedo ${ }^{l}$, Jesús Bravo ${ }^{l}$, Kelly Castrejo ${ }^{l}$, Guillermo Herrera ${ }^{l}$, Mayte Sánchez ${ }^{l}$, Thaily Valencia ${ }^{l}$, Erick Vallester ${ }^{2 *}$ \\ ${ }^{1}$ Licenciatura en Ingeniería Ambiental, Facultad de Ingeniería Civil, Universidad Tecnológica de Panamá; \\ ${ }^{2}$ Facultad de Ingeniería Civil, Universidad Tecnológica de Panamá
}

\begin{abstract}
Resumen En esta evaluación se estudió los coagulantes cloruro férrico y SuperFloc SD 2080, con el objetivo de comparar su efectividad en los procesos de clarificación de agua. Se realizó el estudio con diferentes dosis de coagulante: $3 \mathrm{ml}, 5 \mathrm{ml}, 6 \mathrm{ml}, 7 \mathrm{ml}$, $8 \mathrm{ml}$ y $10 \mathrm{ml}$, sometiéndolas a los procesos de floculación, coagulación y sedimentación. También se realizaron pruebas de jarras, prueba de sedimentación con Cono Imhoff y la prueba de columna de sedimentación. Para determinar la eficiencia de los coagulantes estudiados para la prueba de jarras, las dosis utilizadas fueron: $5 \mathrm{ml}$ por cada dos litros para el cloruro férrico y $6 \mathrm{ml}$ por cada dos litros para el SuperFloc SD2080. Con la prueba de columna de sedimentación, los resultados fueron determinados mediante la curva de porcentaje de isoremoción. La prueba de columna de sedimentación se realizó en dos intervalos de tiempo, en donde los dos primeros intervalos, fueron de cinco minutos y los demás intervalos consiguientes fueron de cada diez minutos hasta llegar a los 120 minutos. Para un tiempo calculado de 57 minutos, los resultados de porcentaje de remoción para el cloruro férrico fueron de $83 \%$ y $37 \%$ para el SuperFloc SD 2080, lo que indica que el coagulante de cloruro férrico fue más eficiente para la remoción de sólidos suspendidos en el agua.
\end{abstract}

Palabras clave Clarificación, coagulación, coagulante, dosis óptima, floculación, índice de lodos, sedimentación.

\begin{abstract}
In this evaluation, the coagulants ferric chloride and SuperFloc SD 2080 were studied, with the aim of comparing their effectiveness in water clarification processes. The study was carried out with different doses of coagulant: $3 \mathrm{ml}, 5 \mathrm{ml}, 6 \mathrm{ml}, 7 \mathrm{ml}, 8$ $\mathrm{ml}$, and $10 \mathrm{ml}$, subjecting them to flocculation, coagulation, and sedimentation processes. Jar tests, Imhoff cone settling tests, and settling column tests were also performed. To determine the efficiency of the coagulants studied for the jar test, the doses used were: $5 \mathrm{ml}$ per two liters for ferric chloride and $6 \mathrm{ml}$ per two liters for the SuperFloc SD2080. With the sedimentation column test, the results were determined by the isoremoval percentage curve. The sedimentation column test was carried out in two-time intervals, where the first two intervals were five minutes and the other intervals consequently were every ten minutes until reaching 120 minutes. By the time of 57 minutes, the percentage removal results of the coagulants were $83 \%$ for ferric chloride and $37 \%$ for the SuperFloc SD 2080, which indicates that the ferric chloride was more efficient for the removal of suspended solids in water.
\end{abstract}

Keywords Clarification, coagulation, coagulant, optimal dose, flocculation, sludge index, sedimentation.

* Corresponding author: erick.vallester@utp.ac.pa

\section{Introducción}

A lo largo de la historia, el agua siempre ha sido una necesidad básica del hombre y de bienestar general en el mejoramiento de la calidad de vida de la población.

Para que el agua pueda ser utilizada para diversas actividades, entre ellas para el consumo humano, es necesario un tratamiento, el cual puede abarcar las fases de coagulación, floculación, sedimentación, etc. [1].

La coagulación es el tratamiento más eficaz para la eliminación de material coloidal en el agua y también puede representar un gasto elevado cuando no se realiza adecuadamente [2].

La floculación es el proceso mediante el cual se juntan las partículas desestabilizadas o coaguladas para formar un aglomerado más grande llamado flóculo y se debe a un mecanismo de formación de puentes químicos o enlaces físicos [2].

La sedimentación de flóculos o decantación es el proceso de depósito de partículas floculentas. Este tipo de sedimentación se presenta en la clarificación de aguas, como 
proceso intermedio entre la coagulación-floculación y la filtración rápida [3].

Los coagulantes más comunes que se usan en el tratamiento de aguas son compuestos inorgánicos de aluminio o hierro como el sulfato de aluminio, aluminato de sodio, sulfato ferroso, sulfato férrico y cloruro férrico [4].

El SuperFloc SD 2080 puede ser utilizado como parte del proceso de coagulación, debido a que ayuda en la sedimentación con coagulantes inorgánicos y orgánicos. También en la clarificación del agua, ya que mejora la calidad del agua del influente y efluente por reducción de sólidos suspendidos y turbidez [5].

El cloruro férrico se utiliza en el tratamiento de aguas residuales y en la producción de agua potable. Es un tratamiento de agua usado debido a su capacidad de reaccionar en el agua a una solución corrosiva y ácida [6].

Su elevado poder de floculación lo destaca como un muy eficiente coagulante en el tratamiento del agua potable y de efluentes sanitarios e industriales, y su habilidad para coagular en un amplio rango de $\mathrm{pH}$ lo convierte en una herramienta de mayor utilidad que otros productos químicos utilizados con los mismos propósitos.

Es especialmente utilizado para la remoción del fósforo y del sulfuro en efluentes tanto industriales como saneamiento [7].

El siguiente artículo se basa en los coagulantes SuperFloc SD 2080 y cloruro férrico $(\mathrm{FeCl} 3)$ con el objetivo de comparar cuál de estos dos es el más efectivo.

\section{Metodología y equipo}

Esta evaluación se llevó a cabo en el laboratorio de Ingeniería Sanitaria de la Universidad Tecnológica de Panamá (UTP).

Para la preparación del agua cruda se pesaron $28 \mathrm{gr}$ de suelo, el cual previamente pasó por un tamiz N. ${ }^{\circ} 200$. Luego en un tanque con $14 \mathrm{~L}$ de agua potable se vertió la muestra de suelo arcilloso, se mezcló por 5 min y se dejó sedimentar durante 15 min para evitar que partículas de arena intervinieran en el proceso de floculación.

La determinación de la dosis óptima se basó en tres pruebas que fueron: prueba de jarras, prueba de sedimentación (Cono Imhoff) y la columna de sedimentación. El gradiente de velocidad se obtuvo mediante el ancho de la paleta $7.5 \mathrm{~cm}$ a 140 RPM por 3 min, 40 RPM por 15 min y 0 RPM por 15 min.

Tabla 1. Gradiente de velocidad

\begin{tabular}{|c|c|c|}
\hline Mezcla rápida & Floculación & Sedimentación \\
\hline $140 \mathrm{RPM}^{130.78 \mathrm{~S}^{-1}}$ & $40 \mathrm{RPM}$ & $0 \mathrm{RPM}$ \\
\hline & $20 \mathrm{~S}^{-1}$ & $0 \mathrm{~S}^{-1}$ \\
\hline
\end{tabular}

\subsection{Determinación de la dosis óptima}

Se realizó mediante la prueba de jarra con el equipo Phipps $\&$ Birds PB-900. Dichas jarras tienen unas dimensiones de 19.5 $\mathrm{cm}$ de altura por $11.3 \mathrm{~cm}$ de base de ancho y la paleta $7.5 \mathrm{~cm}$ ancho de base por $2.5 \mathrm{~cm}$ de alto (figura 1), que simulan los procesos de coagulación, floculación y sedimentación con diferentes dosis de coagulante $(0 \mathrm{ml}, 3 \mathrm{ml}, 5 \mathrm{ml}, 6 \mathrm{ml}, 7 \mathrm{ml}, 10$ $\mathrm{ml}$ ). A través de una comparación visual se determinó que la muestra con la dosis óptima era aquella que contenía los flocs de mayor tamaño y con el espectrofotómetro (HACH DR/2010) se midió la turbiedad según el 2120C Método espectrofotométrico y con el medidor de $\mathrm{pH}$ (OAHUS STARTER 2100) se determinó el pH [8].

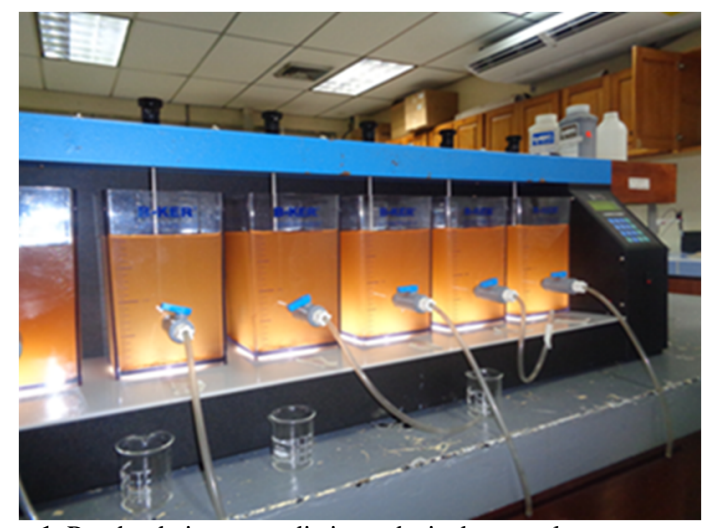

Figura 1. Prueba de jarra con distintas dosis de coagulante.

\subsection{Prueba de sedimentación}

Se utilizó el Cono Imhoff de plástico con las siguientes descripciones: $10 \mathrm{~cm}$ de diámetro y $1 \mathrm{~L}$ de capacidad de volumen.

Para evaluar el índice de volumen de lodos, previamente, se realizó la comparación de las dos dosis más efectivas, que, a nuestro criterio, presentaron mayor concentración de floc sedimentadas en las jarras. Posteriormente se evaluó el índice de volumen de lodo teniendo en cuenta la menor concentración de lodos sedimentadas en los conos.

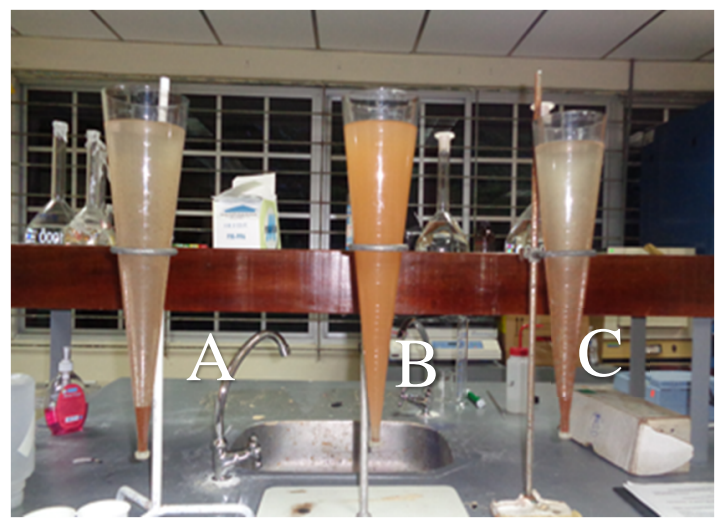

Figura 2. Prueba de sedimentación con coagulante SuperFloc SD 2080 A) Dosis $6 \mathrm{ml}, \mathrm{B}$ ) Agua Cruda, C) Dosis $5 \mathrm{ml}$. 


\subsection{Columna de sedimentación}

Para la prueba de sedimentación tipo II se utilizó la columna de sedimentación de material acrílico con dimensiones de 10 $\mathrm{cm}$ de diámetro y $120 \mathrm{~cm}$ de alto. Se tomaron cuatro muestras simultáneamente, a diferentes alturas $(0.3 \mathrm{~m}, 0.5 \mathrm{~m}, 0.7 \mathrm{~m}, 0.9$ $\mathrm{m})$, en diversos intervalos de tiempos de la siguiente manera: a 0 min, a 5 min y a $10 \mathrm{~min}$ completando $120 \mathrm{~min}$ en total; los datos obtenidos nos ayudaron a determinar el coagulante que remueve más sólidos suspendidos, mediante la curva de porcentaje de isoremoción.

Una vez terminado la medición de la turbiedad (HACH DR/2010) [8], para diversos tiempos y profundidades de ambos coagulantes se procedió a calcular los porcentajes de concentraciones de sólidos suspendidos, por medio de la ecuación 1.

$$
\% S S=\frac{C_{o}-C_{1}}{C_{o}} \times 100 \%
$$

Ecuación 1. Porcentaje de concentración

Fuente: Libro Potabilización del agua de Jairo Alberto Romero Rojas.

Finalmente, se encontró el porcentaje de remoción de ambos coagulantes por medio la ecuación 2 .

$$
\% \text { Remoción }=\frac{\Delta h_{1}}{H} X \frac{R_{1}+R_{2}}{2}+\frac{\Delta h_{2}}{H} X \frac{R_{2}+R_{3}}{2}+\cdots R_{n}+R_{n+1}
$$

Ecuación 2. Porcentaje de remoción.

Fuente: Libro Potabilización del agua de Jairo Alberto Romero Rojas.

\section{Resultados}

Este resultado se obtuvo mediante la comparación de diferentes dosis de coagulantes comunes usados en la clarificación del agua como SuperFloc SD 2080 y cloruro férrico.

\subsection{Dosis del coagulante}

Para ambos coagulantes se determinó la dosis óptima con los valores de turbiedad más bajos de la prueba de jarra (figura 3) y a través de una comparación visual de la muestra que contenía flocs de mayor tamaño.

Observando la gráfica de la figura 3, podemos determinar que el valor más bajo de la turbiedad obtenida del SuperFloc SD 2080 fue de 50 NTU que representa al coagulante agregado en la jarra número 3 , dando como resultado de $5 \mathrm{ml} / 2 \mathrm{~L}$ de dosis óptima.

De igual manera el cloruro férrico fue de 10 NTU que representa al coagulante agregado en la jarra número 4 , dando como resultado de $6 \mathrm{ml} / 2 \mathrm{~L}$ de dosis óptima.

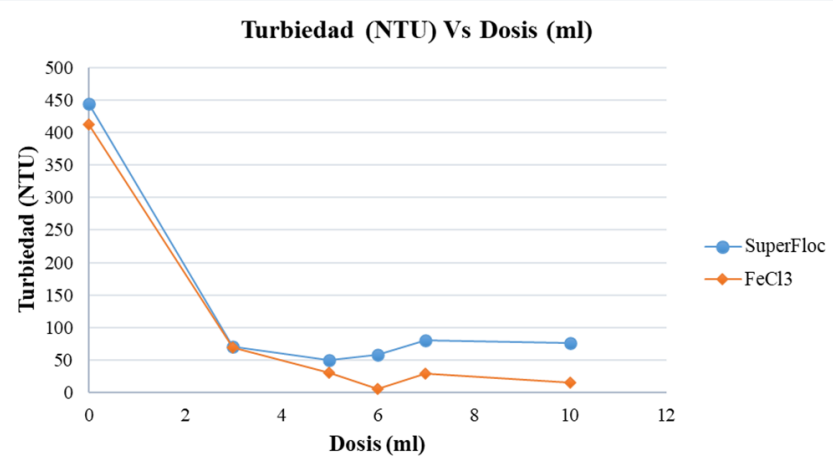

Figura 3. Parámetro de turbiedad.

El índice de volumen de lodos se realizado con Conos Imhoff, para el coagulante de SuperFloc SD 2080 fue que, por cada litro, hay $2.5 \mathrm{ml}$ de sedimento $(1 \mathrm{~L} / 2.5 \mathrm{ml})$. Mientras que para el cloruro férrico por cada litro había $9.0 \mathrm{ml}$ de sedimento (1L/9 ml).

Respecto al porcentaje del lodo en materia, esta dependerá del tipo decantador y coagulantes, estos lodos están formados por las aguas naturales y por sustancias como arcillas, limos o arenas finas.

\subsection{Columna de sedimentación}

Luego que se calcularon los porcentajes de sólidos suspendidos (ecuación 1) del coagulante SuperFloc SD 2080 para cada tiempo y profundidad de la muestra realizada, se elaboró la curva de Isoremoción (figura 4) mediante la carga superficial de $23\left(\mathrm{~m}^{3 *} \mathrm{~m}^{-2 *} \mathrm{~d}^{-1}\right)$ y un tiempo de $57 \mathrm{~min}$, se pudo obtener el porcentaje de remoción (ecuación 2) de $37.00 \%$.

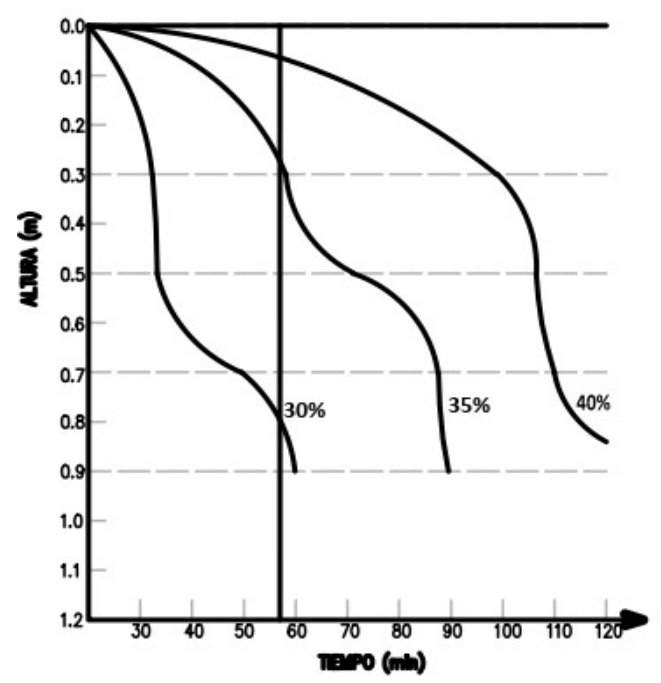

Figura 4. Trayectoria de sedimentación SuperFloc SD2080. 
Igualmente se calcularon los porcentajes de sólidos suspendidos (ecuación 1) del coagulante cloruro férrico para cada tiempo y profundidad de la muestra realizada, se elaboró la curva de isoremoción (figura 4) mediante la carga superficial de $23\left(\mathrm{~m}^{3 *} \mathrm{~m}^{-2 *} \mathrm{~d}^{-1}\right)$ y un tiempo de $57 \mathrm{~min}$, se pudo obtener el porcentaje de remoción (ecuación 2) de $83.00 \%$.

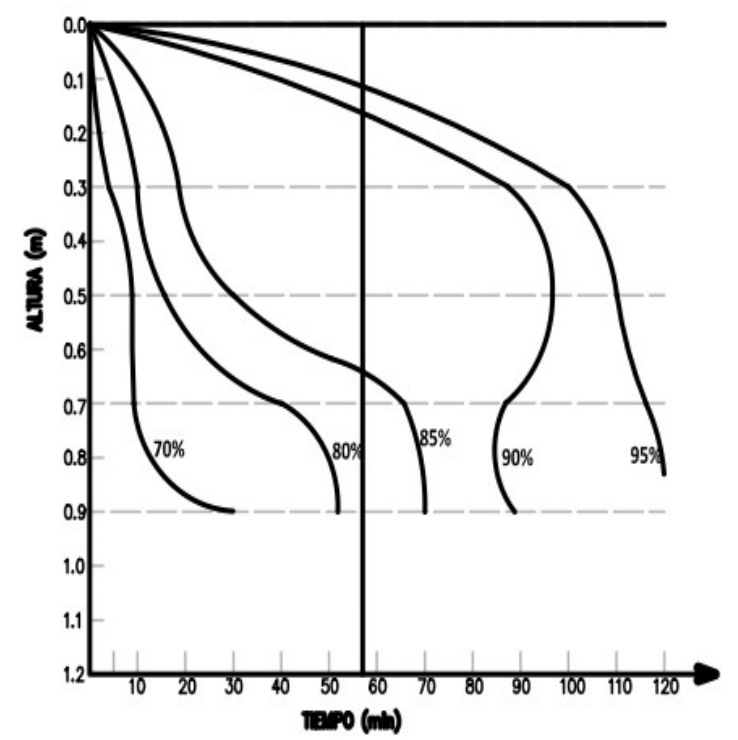

Figura 5. Trayectoria de sedimentación cloruro férrico.

Mediante el cálculo del porcentaje de remoción (ecuación 2) de ambos coagulantes se pudo determinar que el cloruro férrico tiene un mayor porcentaje de remoción de sólidos suspendidos.

\subsection{Parámetros físico-química}

Con estos resultados se realizaron diversos parámetros para determinar la dosis óptima, en la tabla 2 y tabla 3, se observó el agua cruda versus el coagulante cloruro férrico y SuperFloc SD 2080 respectivamente

Tabla 2. Dosis óptima cloruro férrico

\begin{tabular}{|c|c|c|}
\hline Parámetros & $\begin{array}{l}\text { Agua } \\
\text { cruda }\end{array}$ & $\begin{array}{l}\text { Cloruro férrico } \\
(6 \mathrm{~mL} / 2 \mathrm{~L})\end{array}$ \\
\hline Conductividad $(\mu \mathrm{S} / \mathrm{cm})$ & 142 & 156 \\
\hline $\begin{array}{l}\text { Sólidos suspendidos } \\
(\mathrm{mg} / \mathrm{L})\end{array}$ & 289 & 26 \\
\hline $\begin{array}{l}\text { Alcalinidad }(\mathrm{mg} / \mathrm{L} \\
\left.\text { como } \mathrm{CaCO}_{3}\right)\end{array}$ & 58 & 38 \\
\hline Turbiedad (NTU) & 413 & 10 \\
\hline Color & 1350 & 111 \\
\hline $\mathrm{pH}$ & 7.13 & 6.49 \\
\hline $\mathrm{T}\left({ }^{\circ} \mathrm{C}\right)$ & 25 & 24 \\
\hline
\end{tabular}

En la tabla 2 el cloruro férrico a una dosis de $6 \mathrm{ml} / 2 \mathrm{~L}$ se obtuvo un aumento en el parámetro de conductividad, mientras que los demás parámetros sólidos suspendidos, $\mathrm{pH}$, alcalinidad, color y temperatura mostraron una disminución en comparación con el agua cruda.

Tabla 3. Dosis óptima SuperFloc SD 2080

\begin{tabular}{|l|c|c|}
\hline \multicolumn{1}{|c|}{ Parámetros } & $\begin{array}{c}\text { Agua } \\
\text { cruda }\end{array}$ & $\begin{array}{c}\text { SuperFloc SD 2080 } \\
(\mathbf{5} \mathbf{~ m L / 2 ~ L ) ~}\end{array}$ \\
\hline Conductividad $(\mu \mathrm{S} / \mathrm{cm})$ & 143 & 146 \\
\hline $\begin{array}{l}\text { Sólidos suspendidos } \\
(\mathrm{mg} / \mathrm{L})\end{array}$ & 307 & 75 \\
\hline $\begin{array}{l}\text { Alcalinidad }(\mathrm{mg} / \mathrm{L} \\
\left.\text { como } \mathrm{CaCO}_{3}\right)\end{array}$ & 70 & 58 \\
\hline Turbiedad $(\mathrm{NTU})$ & 444 & 50 \\
\hline Color & 1245 & 228 \\
\hline $\mathrm{pH}$ & 7,08 & 6,93 \\
\hline $\mathrm{T}\left({ }^{\circ} \mathrm{C}\right)$ & 23,6 & 24,3 \\
\hline
\end{tabular}

En la tabla 3 el SuperFloc SD 2080 a una dosis de $5 \mathrm{ml} / 2 \mathrm{~L}$ se obtuvo un aumento en el parámetro de conductividad y temperatura, como una disminución en los parámetros de sólidos suspendidos, alcalinidad, turbiedad, color y $\mathrm{pH}$ en comparación con el agua cruda.

\section{Conclusiones}

- Se comprobó que se debe realizar la prueba de jarras, ya que es la más representativa para determinar el comportamiento de los coagulantes en el tratamiento de agua potable para determinar la dosis óptima.

- Se utilizó el parámetro turbiedad, para determinar el porcentaje de remoción, ya que este parámetro fue fácil de medir y no tomaba mucho tiempo.

- Con base en el análisis de los parámetros físico químico, después de la prueba de jarra, utilizando el coagulante de SuperFloc SD 2080 y cloruro férrico, se concluyó que con el cloruro férrico se obtuvieron mejores resultados al momento de realizar las pruebas, ya que ambos lograron remover el material coloidal presentes en el agua cruda.

- La remoción total resultó en un 37\% para SuperFloc SD 2080 y $83 \%$ cloruro férrico, con un tiempo de retención de $57 \mathrm{~min}$ y una carga superfical de $23\left(\mathrm{~m}^{3} . * \mathrm{~m}^{-2} * \mathrm{~d}^{-1)}\right.$. Lo que indica que el coagulante de cloruro férrico es el que tiene mayor porcentaje de remoción de sólidos suspendidos convirtiéndolo en el coagulante óptimo.

\section{Recomendaciones}

- Con base a los valores obtenidos en esta evaluación, el coagulante de cloruro férrico sería óptimo para la 
Vallester (et al): Evaluación de la eficiencia del coagulante SuperFloc SD 2080 frente al cloruro férrico en procesos de clarificación de agua

remoción de partículas en procesos de tratamiento de agua.

- En casos en que el pH esté en un rango ácido, se le puede agregar hidróxido Sódico (soda caustica) para que llegue a un rango base.

\section{AGRADECIMIENTOS}

En la realización y ejecución de esta evaluación participaron personas directas e indirectas que nos ayudaron a realizar este proyecto.

- En primer lugar, al profesor Erick Vallester. Por ser el facilitador principal de esta evaluación, por su orientación y tiempo para el desarrollo de este artículo.

- En segundo lugar, al profesor Cenobio Cárdenas por toda la buena disposición, consejos y orientación en el laboratorio de Sanitaria de la Universidad.

- En tercer lugar, a todos los compañeros que aportaron de su tiempo y conocimiento en esta evaluación.

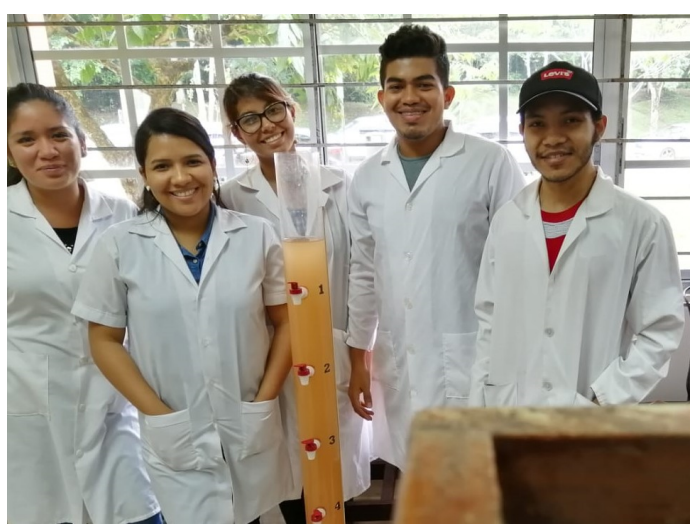

Figura 7. Equipo de trabajo de evaluación.

\section{REFERENCIAS}

[1] Y. A. Cárdenas, «Tratamiento de agua coagulación y floculación,» abril 2000. [En línea]. Available: http://www.sedapal.com.pe/c/document_library/get_file?uuid=2 792d3e3-59b7-4b9e-ae55-56209841d9b8\&groupId=10154.

[Último acceso: 5 junio 2019].

[2] J. M. C. FLÓREZ, «clarificación de aguas usando coagulantes polimerizados: caso del hidroxicloruro de aluminio,» DYNA, vol. 78, nº 165, pp. 18-27, 2011.

[3] I. V. M. Yactayo, «Ingeniero Ambienal.com,» [En línea]. Available: http://www.ingenieroambiental.com/4014/siete.pdf. [Último acceso: 11 junio 2019].

[4] N. C. C. Camacho, «Tratamiento de agua para consumo,» Ulima, no 29, pp. 153-172, 2011.

[5] K. S. SD-2080, «Información Técnica Superfloc,» [En línea]. Available:

https://aniq.org.mx/pqta/pdf/Respaldo/SUPERFLOC\%20SD208 0\%20(L).pdf. [Último acceso: 9 junio 2019].
[6] J. L. Moncayo, «cloruro sodio,» 4 marzo 2019. [En línea]. Available: https://www.clorurosodio.com/cloruro-ferrico/. [Último acceso: 9 junio 2019].

[7] Efice, «Efice buena quimica,» [En línea]. Available: https://www.efice.uy/producto/cloruro-ferrico/\#. [Último acceso: 9 junio 2019].

[8] APHA-AWWA-WPCF, Métodos normalizado para el análisis de agua potable y residual, España: Editorial Díaz de Santos, S.A., 1992. 